\title{
Factores asociados al proceso de matrícula en centros de educación inicial
}

\author{
Factors associated with the registration process on the initial education centers
}

Recibido 12 noviembre 2013 • Aceptado 29 noviembre 2013 • Corregido 11 diciembre 2013

\author{
Julissa Siu Lanzas ${ }^{1}$ \\ Ministerio de Educación Pública \\ San José, Costa Rica \\ yuisiu@yahoo.com
}

\begin{abstract}
Resumen. Este trabajo tiene el propósito de plantear una reflexión en cuanto a las condiciones que conducen a los encargados de las familias a la incorporación de los niños y niñas al primer eslabón del sistema de enseñanza formal, la educación inicial. Se define la posible relación de factores de carácter demográfico, los de índole familiar así como los sociales. Contemplar la relevancia del estudio de cada uno de estos factores, posibilita el mejoramiento sobre la gestión de los centros de educación inicial, ante la inminente presencia de la complejidad multifactorial que deviene en un gran reto para la administración de la educación. Se destaca entre las principales conclusiones que la asistencia a las aulas escolares continúa representando un desafío básico para la dirigencia educativa, incrementado por las particularidades de la divergencia en los contextos sociales, y el manejo efectivo de la información en la planificación directiva, podría convertirse en uno de los efectos correctivos para intentar neutralizar las variables del ambiente, entre los cuales se acentúa la conformación de la familia.
\end{abstract}

Palabras claves. Incorporación; educación inicial; gestión; administración de la educación

Abstract. This paper has the purpose of raising a reflection regarding $f$ the conditions that lead to those responsible for the families to the incorporation of the children to the first link in the system of formal education, initial education. The possible relationship of demographic factors, family nature as well as social ones. Consider the relevance of the study of each of these factors, enables the improvement on the management of the centers of initial education, the imminent presence of multifactorial complexity which becomes a great challenge for the administration of education. Stands out among the main conclusions that school attendance continues to represent a basic challenge to the educational leadership, increased by the particularities of the divergence in social contexts, and the effective management of information in the planning directive, could become one of the corrective effects for trying to neutralize the environmental variables, among which the formation of the family is emphasized.

Keywords. Incorporation; initial education; management; administration of education

1 Máster en Administración Educativa de la Universidad de Costa Rica (UCR), con experiencia en docencia del nivel preescolar en el sector privado y público. Realizó una investigación sobre el comportamiento de la matrícula en centros de educación inicial. 


\section{Contextualización}

La constante trasformación de la sociedad afecta los modelos de organización que evolucionan con rapidez dentro del recuadro global. La información se intercambia con gran dinamismo, por lo que se convierte en un factor elemental tanto para el desarrollo humano como para el de las organizaciones sociales. Castells (1999) destaca, con precisión, las transformaciones sobre el capital social donde "(...) la informacionalización y la globalización son procesos centrales de la constitución de una nueva economía y una nueva sociedad en el cambio de milenio" (p. 4). En un contexto tan variable como el actual, el manejo eficiente de la información resulta en prácticas innovadoras y determinantes en el progreso de las organizaciones como las educativas.

La constitución de la educación en la sociedad del conocimiento, en una confluencia de entornos interculturales, hace manifiesta la evolución consecuente sobre la metodología pedagógica por implementar en las aulas escolares. Tales cambios involucran un proceso educativo con una novedosa perspectiva dinámica para el funcionamiento organizacional. Como garantía de protección sobre los intereses de la comunidad educativa, se requiere de prácticas éticas constantes en el uso de la información -en los mecanismos evaluadores de la calidad del servicio- como validación de una calidad en la enseñanza. La interacción entre diversas disciplinas trasciende los tradicionales métodos mecánicos de intervención educativa, integrando la visión holística del desarrollo humano. Es así como la educación logra revelar las evoluciones sociales, dentro de un accionar con potenciales conexiones entre la diversidad de factores que intervienen en ella. Al considerar la complejidad de los elementos involucrados en el proceso de educación, se posibilita la búsqueda de alternativas para compensar el efecto de fuerzas del ambiente.

Las formas que eligen los individuos para integrar un hogar se han modificado en años recientes. La participación de la mujer en el mercado laboral conduce a la búsqueda de servicios educativos dedicados a la atención de la población infantil, desde que los niños y las niñas se encuentran en una edad temprana. La numerosidad en la totalidad de los miembros de una familia se reduce cada vez más, por diversos motivos. La unión entre las parejas ya ha dejado de ser permanente, por lo cual gran cantidad de mujeres terminan asumiendo la responsabilidad sobre los hijos, de manera independiente. Es así como, la conformación familiar se convierte en un elemento de gran complejidad dentro del proceso de gestión de la educación. La gestión de la educación implica la identificación oportuna de circunstancias por considerar durante el consenso evidenciado en los planes institucionales. La gestión de la educación representa el emprendimiento de acciones sobre una organización visionaria, mediante la concreción de ideas efectivas para conformar un proyecto educativo exitoso, transformando desde el primer paso a cada integrante del centro en un componente esencial para lograr la neutralización de 
fuerzas externas del entorno, las cuales podrían figurar como amenazas para la continuidad de la entidad educativa. Las características poblacionales en cada región conllevan hacia surgimiento de propuestas para favorecer el progreso en cada ámbito educativo.

Las intenciones internacionales enfocadas en el avance de la educación infantil han orientado la gestión hacia cambios visibles en cada sistema educativo. De acuerdo con Sime (2010), el informe del Programa de las Naciones Unidas señala algunos de los avances alcanzados en el ámbito educativo a nivel mundial desde 1990 -aspectos por continuar optimizando en la actualidad -entre los cuales es posible destacar el aumento en la escolaridad de la población y la proporción de matrícula que atienden los centros de educación. Si bien existe una mayor cantidad de individuos alfabetizados, gracias a la expansión de centros escolares en diversas zonas del mundo, la calidad del servicio educativo brindado en ellos se mantiene como un factor por mejorar. A su vez, garantizar el acceso a la educación, requiere del establecimiento de alianzas entre el sector público y privado: como ocurre en la enseñanza infantil. Garantizar la educación desde edades tempranas a la población es traducir en acciones todas aquellas intenciones plasmadas en las propuestas internacionales y acogidas por los planes de gobierno en los diversos contextos de la esfera mundial.

El incremento en la cobertura de los servicios de educación dirigidos a la atención de la niñez ha de planificarse en conjunto con acciones para ofrecer un currículo de calidad, con el cual se pretenda satisfacer las expectativas de cada región tanto como las necesidades de la población infantil. Se trata de asegurar un mayor alcance, que a su vez, vaya acompañado de una práctica pedagógica concentrada en brindar oportunidades para incrementar el potencial de desarrollo humano desde la etapa inicial del sistema formal educativo.

Para garantizar una pedagogía ajustada a las expectativas educativas en cada región, resulta imprescindible ejercer con liderazgo la gestión de los centros de enseñanza, logrando el consenso en cuanto a la misión por emprender entre los colaboradores.

Dirigir los esfuerzos hacia la evolución organizacional, posibilita medidas de planificación focalizadas en el alcance de los objetivos propuestos, que de acuerdo con Mañu (2009), queda expuesto en "el modo en que un centro se organiza, prioriza y enfoca los asuntos para ser capaz de alcanzar la misión, la visión y los valores constituyen la estrategia" (p. 66). Contar con planes estratégicos, elaborados en consenso por los participantes del proceso educativo, demuestra la capacidad de la entidad educativa para establecer el enlace entre la visión hacia la cual proyecta sus objetivos con las acciones correspondientes.

Aprender a conocer, aprender a hacer, aprender a vivir y aprender a ser, pilares la educación propuestos por UNESCO desde 1996, continúan con vigencia para la gestión de los centros de enseñanza. Si bien interesa ratificar una extensa cobertura en cuanto a la educación de la 
población, se requiere, en igual medida, de acciones administrativas y pedagógicas en lectura con las exigencias de cada ambiente. Traducir en acciones las iniciativas de mejora del proceso educativo resulta posible a través de liderazgo y la planificación dirigida hacia el cambio de la situación actual dentro de las condiciones de cada contexto. Las propuestas nacionales reflejan en alguna medida las intenciones de los organismos exteriores dedicados a la atención de los intereses de la población infantil.

Corresponde a una nación, asumir con responsabilidad la gestión sobre todas aquellas propuestas orientadas hacia el desarrollo de la enseñanza infantil. Los centros de educación pueden llegar a convertirse en intérpretes de transformaciones didácticas propuestas a nivel de cada nación, para favorecer en la construcción de un proyecto de avance social, donde se promueve la conformación de organizaciones competitivas identificadas con los intereses del entorno. Sin embargo, en numerosas ocasiones las propuestas han quedado sin efecto, debido al exceso en la elaboración de documentos de planificación que terminan por acumularse con una deuda en su implementación.

En la búsqueda por ofrecer un servicio de accesible a la población costarricense se organizan operaciones dirigidas a fortalecer el sistema de educación, a través de la expansión en la cobertura, así como con la apertura de centros de atención en sectores en riesgo social, entre ellos los Centros de Nutrición (CEN), Centros de Atención Integral (CAI) y redes de cuido (CECUDI) en coordinación con el Ministerio de Salud. Estas propuestas contienen intenciones que pretenden favorecer el proceso de desarrollo infantil, a nivel educativo resultan opciones que inadvertidamente intervienen en la aparición de cierta brecha entre el interés didáctico del ciclo de enseñanza inicial y su aplicación, dentro de la normativa frecuente de ese tipo de centros.

Acerca de la trascendencia de la educación inicial para el desarrollo del país, se puede destacar la labor pedagógica enfocada en mediar entre el currículo didáctico y las expectativas de los beneficiarios, para construir un proyecto de gran alcance para la sociedad. La expansión en la cobertura del sistema educativo trae consigo la ampliación en la complejidad organizacional por gestionar. Las demandas sociales en cada contexto, representan un desafío de sumo compromiso para la gestión educativa.

\section{Condiciones sociales emergentes}

La concreción de acciones correspondientes con las intenciones internacionales, integran planes a nivel de región que definen una ruta por emprender a través de una gestión de la educación ajustada a las particularidades del contexto. Las condiciones dentro del sistema de educación, en relación con las características demográficas y familiares, pueden ser contempladas como parte de un fenómeno por comprender. 
Más allá de la justificación axiomática por brindar servicios de educación inicial básicos para el desarrollo infantil integral, se han de cimentar las bases de un sistema de calidad. De acuerdo con las proyecciones del Ministerio de Educación Pública (MEP) sobre la cobertura de la educación de la niñez desde los cuatro años y medio de edad, se ha esperado llegar a cerca de un $72 \%$ de alcance para el 2015 como parte del Plan de Acción a nivel nacional (MEP, 2002). El impacto de la educación en los diversos contextos del país puede llegar a representar beneficios a nivel social para el futuro desarrollo de la nación, siempre que se contemple en condiciones equitativas el acceso a la actividad educativa en cada zona. El alcance de la planificación nacional debe ir en concordancia con las condiciones sociales de la región, donde se trate de asegurar una expansión estratégica que responda a las proyecciones demográficas del territorio. En determinadas comunidades, podría decirse que, la dotación de servicios de transporte público, pudo haber sido la prioridad ante la edificación de centros educativos con distancias reducidas entre ellas.

En distintos contextos el reto para la gestión de la educación podría quedar evidenciado en el mantenimiento activo del estudiantado en los salones escolares. En datos estadísticos del MEP (2002) es posible detectar un determinado porcentaje de estudiantes que desertan de los centros de educación formal, en su mayoría del ciclo interactivo II. Entre las posibles causas por las cuales los niños o niñas se ausentan se pueden mencionar la inestabilidad laboral de sus encargados, en muchos casos ligados a los movimientos migratorios -dentro de la misma zona del país como hacia otras naciones de Centroamérica- asociados con la condición socioeconómica de los involucrados. Al mismo tiempo, aparece una representatividad de ese ciclo educativo inferior en proporción a la asistencia del siguiente nivel preescolar de transición, el cual en similar medida, resulta menor a la totalidad de matrícula en el primer curso de primaria. Las estadísticas reflejan la necesidad de atender a la población infantil mediante una gestión que considere la situación de los distintos núcleos familiares y la valoración que estos realizan sobre la posibilidad de ingresar al sistema de educación inicial. Novedosos consensos en la política se precisan en la formulación de gestiones que favorezcan a la mayoría de los beneficiarios de los servicios educativos.

Emplear la información de manera operacional para los objetivos organizacionales, genera una continua reflexión y análisis en los centros de educación. En referencia a la ampliación del potencial organizacional para concebir cambios, Fernández (2005) comprende que "la gestión del conocimiento, como se ha dicho, consiste en acumular experiencia colectiva sobre problemas y oportunidades que surgen en la operativa cotidiana de las organizaciones" (p. 222). Gestionar con liderazgo desde la dirección escolar convierte en causa común la generación de conocimiento dentro del proceder de la cultura organizacional, como muestra de competencias de adaptación y sensibilidad ante las necesidades del contexto en el que se inserta la institución. 
La consideración de los datos poblacionales así como sus proyecciones, evidencian la necesidad de emprender desde la gestión de la educación un análisis sobre las actuales condiciones de apertura y continuidad de los servicios de educación inicial con respecto a la realidad de las comunidades educativas.

De acuerdo con las estadísticas del Instituto Nacional de Estadística y Censo (INEC, 2012), la totalidad de nacimientos en Costa Rica se ha reducido en ciertos sectores del territorio. Ello podría estar relacionado con cambios sociales en la población en edad reproductiva, como las metas ocupacionales, la accesibilidad en los métodos de control natal y los movimientos migratorios. En zonas periféricas del país la cantidad de nacimientos ha estado en aumento, mientras que en las céntricas se aprecia una reproducción con tendencia a la disminución, tal y como se visualiza en la tabla 1.

\section{Tabla 1}

Nacimientos en las provincias según la residencia de la madre 2002 y 2012

\begin{tabular}{|l|c|c|}
\hline \multicolumn{1}{|c|}{ Provincia de residencia de la madre } & Total nacimientos 2002 & Total nacimientos 2012 \\
\hline & & \\
\hline San José & 24165 & 22619 \\
\hline Alajuela & 13632 & 14658 \\
\hline Cartago & 7262 & 7365 \\
\hline Heredia & 6227 & 6623 \\
\hline Guanacaste & 5078 & 5955 \\
\hline Puntarenas & 7301 & 7674 \\
\hline Limón & 7479 & 8432 \\
\hline
\end{tabular}

Fuente: Datos de población preliminares del INEC, 2012.

La cantidad de niños y niñas en edad para ingresar en los centros de educación preescolar puede estar afectada, en algún grado, por los índices de mortalidad infantil de cada provincia así como por el efecto de migraciones en la región interna y las internacionales. Emerge la oportunidad de proponer acciones efectivas, con el propósito de dirigir cada esfuerzo hacia la implementación de medidas socio-educativas pertinentes, en las cuales se refleje una visión estratégica del escenario por gestionar. 
Para el contexto nacional, los niveles de natalidad así como de fecundidad tienden a la reducción, por distintos motivos. Ante tal panorama, las expectativas especulativas sobre la conformación de agrupaciones de infantes densas por salón, pretenden una aspiración hacia un estado antiguo, de incierto regreso y de exiguo beneficio para brindar la atención demandada por los niños en edad temprana.

Similar a las tasas de natalidad que han estado reduciéndose a nivel nacional, las mujeres se conservan dentro de porcentajes de fecundidad bajos. El promedio de hijos o hijas se encuentra determinado en dos o tres por mujer, mientras se proyecta al descenso en los próximos años, a uno o dos por familia, de acuerdo con los datos de proyección del INEC (2012).

Por las transformaciones sociales que están transcurriendo resulta evidente la necesidad de fundamentar en forma sistemática, a través de un análisis que ofrezca insumos, con el propósito de propiciar la reflexión de los funcionarios encargados de las políticas educativas, para contemplar desde la gestión de la educación la apertura hacia la promoción de ajustes en el sistema inicial debido a los fenómenos demográficos así como otros factores que podrían ejercer influencia en el comportamiento de la matrícula en los centros de educación infantil.

Resulta imprescindible contar con información fundamentada en los escenarios de acción de los agentes del proceso de educación, a partir de la cual sea posible guiar el emprendimiento de decisiones desde las que se logre ajustar los servicios dirigidos a la población infantil, con base en las circunstancias sociales - familiares, demográficas e institucionales - con el propósito de cumplir con la misión de compensar las demandas de la sociedad actual.

La planificación administrativa estratégica en el ámbito educativo prevé los emergentes elementos que podrían ejercer fuerzas amenazantes contra el esperado proceso de educación; campo de acción de la administración de la educación según se explicita seguidamente.

\section{Administración de la educación y la organización educativa}

Ante los recientes cambios sociales y demográficos, la administración de la educación posibilita la previsión del recurso humano como también de las diversas herramientas técnicas fundamentales para desplegar una educación de calidad.

Como fuente de una antítesis de las tradicionales prácticas administrativas de contingencia promovidas desde la improvisación, se requiere ante los contextos complejos del ámbito de acción educativo, de líderes comprometidos con los apremiantes cambios constantes de las características en cada comunidad educativa, en donde se promueva un ejercicio pedagógico en función del desarrollo integral del estudiantado. El carácter del líder encargado de la organización educativa contiene la capacidad para analizar y sintetizar información, así como 
la maestría de identificar las habilidades de los colaboradores y coordinar equipos fortalecidos para atender de manera oportuna las necesidades de la comunidad.

Si bien la especialización de los profesionales en el campo de la administración de la educación requiere de prácticas técnicas tradicionales, en el perfil esencial del directivo educativo destaca la anuencia para resolver las situaciones que surgen con efectividad.

Quienes se encargan de administrar desde puestos directivos adquieren la responsabilidad de organizar, coordinar y evaluar todo proyecto por implementar en la organización educativa, a partir de las particularidades e intereses de aquellos que son parte del proceso de educación. Por consiguiente, la administración debe considerar con especial observación las distinciones de la región en donde se inserta el centro infantil para ofrecer el servicio requerido por los beneficiarios. Resulta imprescindible comprender una visión holística, en cuanto a la interacción de variables del ambiente que intervienen en todo fenómeno educativo.

Administrar para la mejora de los centros educativos resulta en la proyección de la labor educativa hacia la comunidad, con el propósito de lograr la identificación de los agentes con la responsabilidad social, lo cual posibilita la mediación entre los requerimientos del contexto con las metas de la organización, como González (2011), lo determina expresando:

Esta posibilidad de interrelacionar la institución con el entorno permite incorporar a la institución una función no muy reconocida y explotada cual es la de mediación, es el adentrarse en el complejo y rico mundo de significados subjetivos donde los individuos de dentro y de fuera de la institución, que interactúan, desarrollan y dan sentido a las prácticas cotidianas. (p. 296)

Como organización, el centro de educación emprende un compromiso con la comunidad hacia la cual sirve, a partir de la estructura gestionada por la directiva educativa. Actuar de forma aislada a las problemáticas familiares y sociales del contexto por dirigir, demuestra la perspectiva arbitraria desde la cual se tiende a perseguir un nivel deseable o superficial de desempeño. Conviene más la reflexión y valoración de las condiciones en las que se administra, en búsqueda de una apreciación más integral en cuanto a los fenómenos educativos.

El efecto recíproco entre los cambiantes fenómenos sociales y los complejos sistemas educativos adquiere un matiz distinto durante la época actual, debido al incremento en la variabilidad constante de los elementos que intervienen en tales procesos de la sociedad.

Cada administración en las instituciones de educación infantil asume un compromiso con la responsabilidad correspondiente de considerar las particularidades del escenario de actuación para beneficio de los usuarios, incluyendo a estudiantes y padres que conforman la comunidad educativa. La gestión de organizaciones educativas sobrepasa la habitual 
tramitación burócrata, para emprender acciones por medio de las cuales se beneficie a todos los implicados en el proceso de educación.

Para gestionar la superación de la organización educativa, existe el consenso durante la planificación en cuanto a las necesidades prioritarias por solucionar. "La gestión escolar está dirigida no sólo a los aspectos técnico-administrativos, sino también a la sensibilización y orientación de la comunidad escolar" (Pérez, 2002, p. 28).

La proactiva participación en la planificación estratégica del proyecto educativo del plantel institucional, de representantes de las familias y de determinados integrantes de la comunidad, se precisa durante la premisa de la misión por emprender.

Todo proyecto de enseñanza en las organizaciones infantiles, avanza hacia el mejoramiento del servicio educativo, a partir del liderazgo que ejercen los líderes en cada centro de educación, con el propósito de generar espacios de interacción favorables para el logro de una labor condensada en un equipo estructural comprometido con las metas comunes.

La pérdida de una visión precisa de la función última de la administración de la educación - determinada en el estudiantado y su aprendizaje- conlleva una insuficiente identificación entre las actividades realizadas en los centros educativos con un auténtico desarrollo infantil. La capacidad para transformar la misión organizacional hacia la cual se dirigen los esfuerzos de los participantes del proceso educativo, emerge de un liderazgo dinámico influyente sobre todo ámbito asociado con la enseñanza. Repetir las antiguas formas de dirigir la institución educativa, donde prevalece la perspectiva conductista sobre el desarrollo curricular, conlleva al tropiezo con consecuencias que comprometen el aprendizaje de los involucrados en el proceso de educación y a una posible confusión de los beneficiarios, en cuanto a la coherencia del servicio por demandar con las características de la población infantil.

La habitual tramitología administrativa, ha representado un concreto distractor de interferencia entre la gestión dinámica de los ambientes educativos y la rutinaria reproducción de prácticas arbitrarias de dirección en las organizaciones al servicio de la educación. Corresponde generar propuestas pedagógicas atractivas para la comunidad, de manera que se logre interesar a la población en la oferta de cada centro.

Si bien los procedimientos administrativos en cualquier empresa implican el despliegue periódico de técnicas diagnósticas para determinar los puntos sensibles de la compañía y emprender acciones correctivas, la opinión de quienes dirigen, en cuanto a la aplicación de métodos similares en el área educativa, sostiene su aparente irrelevancia ante la complejidad de los contextos. Cuando la gestión de los centros de enseñanza parte de un diagnóstico a través del cual se evidencien las debilidades de la organización así como las amenazas provenientes del ambiente por neutralizar, la planificación refleja una perspectiva consensuada durante la 
elaboración de proyectos de mejora, de manera que se evita la tendencia hacia la ejecución de acciones administrativas improvisadas.

De efectuar con responsabilidad el seguimiento de las actividades ejecutadas en cada período lectivo, es viable proponer mayores metas organizacionales, a través de las cuales se generen perspectivas y prácticas innovadoras, con el propósito de asegurar a los beneficiarios un mejor servicio educativo.

Una auténtica planificación visionaria, intenta acortar con cada acción el trayecto hacia la culminación de metas organizacionales a largo plazo, valiéndose de propuestas innovadoras ante la incertidumbre que proviene de los elementos del entorno por gestionar. Desde toda acción, proyecto, plan y programa institucional, se proyecta la visión hacia donde se realiza cada esfuerzo de los integrantes del centro educativo, sobre un estilo de innovación continua que conduzca al progreso organizacional. En función de las políticas educativas vigentes, se establece la elaboración de proyectos a nivel de cada organización, como guía para la gestión de recursos administrados hacia el progreso en la oferta educativa.

Romero (2004) da a la innovación dentro del proyecto de educación un sentido de proceso basado en la experiencia personal y de conformación colectiva, "como un compromiso de toda la organización en el que la creación de nuevo conocimiento no consiste sólo en aprender de otros o en adquirir conocimiento del exterior, sino que demanda una interacción intensa y laboriosa de todos" (p. 132). La innovación educativa genera la proyección de acciones propuestas desde la labor de equipo del plantel organizacional, con el propósito de fortalecer el currículum ofrecido a la comunidad con la concreción de intenciones comprometidas con el aprendizaje.

Se advierte así la trascendencia del desarrollo de prácticas, a través de las que se pretenda gestionar acciones relevantes para el mejoramiento organizacional, con base en la formulación e implementación de proyectos institucionales por medio de los cuales se coordinen dinámicas con agentes comprometidos con la misión educativa. Los centros educativos con una considerable afluencia de beneficiarios, son aquellos donde año tras año se realizan propuestas de interés para la comunidad educativa, institucionalizando de esa manera el desarrollo de proyectos atractivos y funcionales para el servicio a la región.

Cerro (2005) advierte como la elección de la excelencia en la gestión del centro educativo considera elementos favorecedores y obstaculizadores del contexto por dirigir. Los modelos adaptados para gestionar con alto nivel dentro del ámbito educativo se saben de originen empresarial y consiguen constituir una posibilidad para administrar mejoras oportunas en los procesos pedagógicos, puesto que se parte del análisis fundamentado en datos reflejantes de la realidad los cuales admiten la discusión sobre las temáticas relevantes para contemplar 
las decisiones por precisar. La generación de los cambios demandados comienza por actuar en consecución de los objetivos propuestos con actitud emprendedora, con el propósito de avanzar en equipo hacia el progreso organizacional.

Con el propósito de designar los recursos requeridos por cada región educativa durante un ciclo lectivo, el Ministerio de Educación Pública costarricense establece la normativa en referencia a la programación del nivel preescolar, bajo la cual se ampara la continuidad de la asistencia educativa en las diversas localidades del país, de acceso privado y público.

\section{Programación del servicio preescolar}

En Costa Rica la distribución estudiantil que se programa periódicamente en el sistema de educación nacional se basa en rangos estandarizados para las zonas rurales y urbanas del país para atender las necesidades de cada comunidad con base en las particularidades de áreas céntricas y periféricas, al ser estas últimas más sensibles a las limitantes en recursos como el transporte público.

Para ingresar al sistema nacional de educación preescolar, se definen los cuatro años y seis meses, en el ámbito público, ubicando a los niños en el grupo interactivo Il del ciclo materno infantil. El ingreso para el siguiente nivel inicial queda establecido a los cinco años y seis meses y coloca a los estudiantes en el ciclo de transición, previo a la iniciación escolar. Ingresan al mismo tiempo en ambos ciclos educativos - de contar con capacidad locativa- los chicos que a la segunda semana del mes de febrero del curso lectivo han cumplido cuatro años o cinco años tres meses.

En códigos específicos de Interactivo Il se requieren 20 estudiantes por aula como mínimo para autorizar la apertura así como la continuidad del servicio en centros ubicados en zonas urbanas y 15 en jardines del área rural. En el ciclo de transición, el MEP solicita a las instituciones educativas urbanas un mínimo de 25 niños o niñas y de 20 en las rurales. De existir capacidad locativa en cada jardín se posibilita la inscripción de hasta 35 estudiantes en las salas con código específico de transición y 25 niños en aulas interactivo II, en zonas urbanas. Esas condicionantes se han mantenido en dichos rangos por varios años, olvidando contemplar la posibilidad de incluir la docencia asistencial para la atención de grupos de infantes, por lo que se designa a una única educadora por sección.

En zonas rurales se habilita la apertura de salones para la atención de niños y niñas en condición etaria para el ingreso a interactivo II y para transición, en agrupaciones mixtas determinadas como grupos heterogéneos, en los cuales se recibe a un rango entre 10 y 15 estudiantes. 
La prestación de servicios en dos centros educativos durante un mismo período lectivo se ha instalado como código itinerante en el ciclo de transición, mientras se cumpla con la condición de factible acceso entre las comunidades donde se ubiquen ambos centros y tener personal especializado. La suma de matrícula entre los grupos de los dos centros debe llegar a un mínimo de veinte estudiantes, sin embargo, la autorización de nuevos códigos de este tipo se ha suspendido.

Corresponde a la administración del centro educativo con interés por la apertura de secciones preescolares, poseer la siguiente agrupación del alumnado descrita para las diversas zonas nacionales, tanto como la designación de códigos correspondiente en la institución infantil tal y como se indica en la tabla 2.

Tabla 2

Cantidad mínima de estudiantes por grupo en el período inicial (Distribución inicial por año lectivo)

\begin{tabular}{lccc}
\hline Prestación del servicio & $\begin{array}{c}\text { Materno y } \\
\text { transición } \\
\text { Urbano }\end{array}$ & $\begin{array}{c}\text { Materno y transición } \\
\text { Rural }\end{array}$ & $\begin{array}{c}\text { Heterogéneo } \\
\text { Rural }\end{array}$ \\
\hline $\begin{array}{l}\text { Código específico } \\
\text { Recargo de funciones } \\
\text { para centros que tienen } \\
\text { código específico }\end{array}$ & $20-25$ & $15-20$ & $10-15$ \\
$\begin{array}{l}\text { Recargo de funciones } \\
\text { para centros que no } \\
\text { tienen código específico }\end{array}$ & $26-36$ & $21-31$ & - \\
\hline
\end{tabular}

Fuente: Normativa del MEP, 2009.

\section{Componentes institucionales del servicio preescolar.}

\section{Estructura de las organizaciones.}

La educación preescolar comprende la interacción de factores como la estructura organizacional que adquieren un matiz específico por su valoración individual o por la magnitud producida en su enlace con otros elementos tal y como los expone Maureira (2006):

(...) si el desafío de los sistemas educativos es incrementar su calidad constantemente fortaleciendo la autonomía y participación en los centros de sus actores para mejorar los 
aprendizajes, los factores clave que contribuyen a ésta, cualquiera sea su fundamento, están fuertemente imbricados por una serie de factores interrelacionados en diferentes niveles de la organización escolar. (p. 8)

Al respecto, se sabe sobre otros componentes organizacionales como la distribución temporal y la metodología pedagógica, todos igual de importantes. Los elementos mencionados intervienen en grados específicos para mantener la creciente demanda de los servicios educativos y es fundamental su consideración desde la administración de la educación.

A su vez, es importante considerar que los centros escolares mantienen determinadas formas de interacción entre sus integrantes para la conformación de proyectos organizacionales definiendo roles concretos por desempeñar en el alcance de los propósitos, como queda expuesto por Robbins y Coulter (2000):

Esa estructura puede ser abierta y flexible, sin una delineación clara y precisa de los deberes de cada empleado ni una adhesión estricta a distribuciones explícitas del trabajo (en otras palabras, una simple red de relaciones de trabajo informales), o la estructura puede ser más tradicional, con reglas, reglamentos y descripciones de puestos clara y cuidadosamente definidos, identificando a algunos miembros como "jefes", los cuales tienen autoridad sobre otros miembros. (p. 5)

Ante la complejidad de los contextos por administrar en el ámbito educativo, emergen estructuras abiertas con una mayor capacidad autónoma para responder de manera oportuna a las situaciones problemáticas del entorno. Las condiciones familiares y sociales agregan incertidumbre en la planificación de las actividades por desarrollar como proyecto educativo.

\section{Distribución temporal.}

La estructura de las organizaciones educativas se encuentra en un proceso de ajuste ante las emergentes transformaciones sociales. Sobre estos elementos "destacan la importancia de llevar adelante enfoques de aprendizaje en situaciones donde, más allá de las acciones rutinarias, deben crearse condiciones de donde puedan surgir, en el futuro, mejores formas de decidir y accionar" (Simonetta, 2010, p. 8). Mantener una actitud pasiva en respuesta a las particularidades del constante cambio del entorno, puede conducir a actuar de manera tradicional cuando se requiere de dinamismo y acciones efectivas en la práctica pedagógica. Construir la identidad de la organización a través de una misión consensuada en la planificación institucional requiere de decisiones oportunas coherentes con las demandas de cada contexto educativo. Las jornadas lectivas pueden adaptarse con innovación a las características de la población infantil, por medio de la promoción de encuentros atractivos que sobrepasen la rutina habitual, para provocar la creciente solicitud de los servicios del centro educativo. 


\section{Metodología de educación infantil.}

Los programas de estudio para los niveles de educación preescolar han permanecido intactos desde su primera implementación. Si bien ambos tienden al énfasis en acciones desde las cuales se promueva la construcción de conocimientos en interacción con los compañeros y el ambiente, con la guía docente, al menos en su planteamiento teórico, en la práctica de los salones de clases persisten las actividades academicistas en donde el estudiante reproduce temáticas escolares mediante técnicas mecánicas tradicionales. Es así como Kontos y Dunn, citados por Ruiz (2002), definen a la programación de la educación inicial orientada hacia el alcance de la calidad, en correspondencia con el modelo educativo constructivista, centrado en la promoción del aprendizaje activo y la exploración. El período de enseñanza preescolar resulta crítico para construir las conexiones mentales de aprovechamiento motor requeridas en las siguientes etapas del sistema de primaria, por lo cual es trascendental proveer de experiencias concretas y personalizadas a los grupos de infantes en los jardines.

El análisis en cuanto a las problemáticas con cierto grado de incidencia sobre el comportamiento de la matrícula en los centros de educación inicial, a través de una visión globalizadora, comprende asimismo de las condiciones institucionales, como de aspectos demográficos, familiares y sociales.

\section{Aspectos demográficos a nivel regional}

La composición familiar ha pasado de la estable relación nuclear a otras tipologías como las parejas cohabitantes y el creciente estado monoparental. Esa variación sobre las condiciones familiares va acompañada de transformaciones en los contextos laborales, para admitir la incorporación femenina en el mercado. El costo de reproducción puede representar, para los individuos, un aspecto determinante para suponer el compromiso con un extenso conjunto de miembros en el hogar. Ante esta coyuntura, se origina la percepción de una reducida fecundidad de la población.

Intervienen conjuntamente distintos elementos asociados con los movimientos migratorios de la región -internos y al exterior- así como la depreciación en la fecundidad durante la condición etaria reproductiva y los índices de natalidad, los cuales pueden llegar a definir la demografía nacional. Tales condiciones han de ser abordadas desde la dirección escolar, de manera que sea posible adoptar una visión particular con el propósito de intentar compensar en alguna medida los factores ambientales en el proyecto educativo en las distintas regiones del país.

Dentro de las condiciones demográficas, la fecundidad adquiere relevancia en el sentido de las proyecciones en cuanto a distribución del costo sobre los recursos pertinentes para 
las distintas comunidades educativas. La depreciación de fecundidad entre las habitantes, es un elemento de trascendencia para la planificación en los sectores nacionales: como el de educación.

Las relaciones entre las parejas parecen tener un carácter cada vez más transitorio, y ello conduce a los individuos a tomar decisiones contingentes ante los cambios en su condición civil. Asimismo, el acceso a los recursos - por influencia de la propia circunstancia natural o bien por el estado laboral - transforma la perspectiva femenina en cuanto a su disposición para considerar el aumento en el tamaño del núcleo familiar.

En la sociedad costarricense, la mujer ha demostrado un mayor impulso para incorporarse dentro del mercado laboral, conforme se avanza en el presente siglo. Sin embargo, la remuneración destinada a la participación masculina supera a las expectativas que podría aspirar su contraparte. El género femenino continúa bajo cierta dependencia socioeconómica de la pareja, de la solidaridad de familiares o de programas de asistencia social, lo cual define el posible interés por fecundar durante su período reproductivo.

En concordancia, la vida reproductiva tiende a representar una etapa controlada para las mujeres, en especial para aquellas con diversas aspiraciones académicas y profesionales. La condición etariaóptima para la vida reproductiva en la naturaleza femenina, en diversos contextos del país ha sido influenciada por los efectos sociales de la desigualdad de oportunidades para incorporarse en el mercado laboral, entre ellos una brecha entre las políticas nacionales de asistencia y su aplicabilidad, durante el período comprendido desde el embarazo y los primeros seis años de existencia de los hijos. El costo de reproducción para asumir la responsabilidad por la descendencia familiar, representa un aspecto sensible para la mujer con diferentes intenciones personales, como las académicas.

El control sobre la fecundidad en la vida reproductiva femenina lleva a revelar niveles de natalidad que se han reducido en los últimos años. Los índices en cuanto a la totalidad de nacimientos en la zona se han encontrado en descenso y con tendencia a la estabilización. Si bien la población femenina durante su vida reproductiva se proyecta en los siguientes años con una presencia constante, la cantidad de hijos por mujer tiende a reducirse, y se estima puede convertirse a menos de dos niños por hogar. Como afirma Rosero-Bixby y Jiménez (2012), "la proporción de niños y jóvenes en la población está en franca caída y continuará disminuyendo en el futuro" (p. 56).

\section{Condiciones familiares de la comunidad educativa}

La estabilidad en la composición familiar se ve afectada por los retos personales asumidos por la mujer, así como por la dependencia de la madre, al menos en el sentido económico, que 
de manera ideal ha de ser asumida por el responsable por la paternidad de la descendencia. Esa variación sobre las condiciones familiares revela la adaptación a transitorios tipos de familia, lo cual se acompaña de transformaciones a nivel laboral.

La influencia dogmática tradicional e idealizada en diversas zonas de Latinoamérica continúa ejerciendo un fuerte impacto sobre uno de los roles definidos para la mujer en la sociedad, encargada de asegurar la concepción familiar como: la reproductora maternal. Para Pedone (2006), "el varón aparece como quien debe cumplir con el rol de sostén económico de la familia y la mujer como el miembro que debe organizar, gestionar y asegurar la reproducción social del grupo doméstico" (p. 156).

Con la incorporación de la fémina en otros ámbitos sociales de acuerdo con determinadas condiciones estatales, se van adquiriendo nuevas cargas emocionales y sobre el capital financiero, provocando variaciones en cuanto a las uniones conyugales, a lo cual se refieren Quilodrán y Castro (2009), cuando mencionan que "las especificidades en la formación de las parejas y su disolución por países, grupos sociales, características socioeconómicas y residenciales persisten, pero ocurren a distintos ritmos configurando escenarios familiares cada vez más variados" ( $p$. 283). La tradición consolidada en la familia nuclear, conformada por el padre, la madre y el hijo que comparten bajo un mismo hogar, ha mutado hacia diversas formas adaptativas ante la ambivalencia del individuo y su circunstancia. La elección informal de parejas cohabitantes o uniones consensuales ha cobrado gran representatividad en distintos estratos sociales. Emerge a su vez, como consecuencia de la fragilidad en los términos de las actuales filiaciones, la paternidad ausente figurada en el acentuado aumento de los hogares monoparentales.

Lajefatura femenina en el hogar se traduce, a su vez, en patrones de interacción que modifican la dinámica familiar. Los espacios de convivencia entre los integrantes de una familia han estado transformándose por las exigencias laborales adquiridas por los encargados del hogar así como por los distractores tecnológicos dirigidos a la juventud. La figura paterna se asocia con la del proveedor y, si bien la madre ha asumido en similar medida ese rol aunado a las responsabilidades familiares como la atención de los hijos y el mantenimiento del hogar, la intensidad con la cual se manifiesta en el nivel productivo ha sido menor que la del hombre. En distintos contextos los hermanos mayores cuidan de los menores además de cumplir con las asignaciones escolares. La familia ha cambiado el parque infantil por la adquisición de juegos didácticos, ha aumentado la frecuencia para presionar a los infantes en la consecución de propósitos académicos y se han dejado de compartir historias por atender la programación televisiva.

Las tradiciones familiares, descansan en un pasado idealizado, que provoca un impacto evidente sobre la definición de los roles en la sociedad, la cual por un lado, exige la reproducción rutinaria de valores, y por otro, demanda gran parte del tiempo de los encargados de las familias para tareas del mercado. De tal forma, la composición familiar se precisa de manera confusa 
entre los adultos y proyecta en diversos contextos una imagen caótica de la interacción social en los niños.

La labor doméstica forma parte del desempeño femenino, mientras el rol masculino se encarga de proveer ingresos para la familia, en las uniones matrimoniales de carácter tradicional o nuclear. La presencia de descendencia aparece como una limitante, en alguna medida, de la participación de las madres en la producción del mercado de trabajo. La madre se enfoca en la atención de la actividad del hogar, en numerosas ocasiones, de manera independiente y sin contar con el respaldo económico o emocional de la pareja. Se suele recurrir al apoyo de los abuelos o de los tíos de los niños y niñas, para cumplir con los requerimientos del cuidado socioeducativo infantil.

La desintegración en el núcleo familiar y el aparente desinterés de quienes se encargan de los infantes delega en el sistema de educación formal, la responsabilidad por el desarrollo de la niñez.

El compromiso con la educación infantil recibe una valoración primordial por la familia, de forma coherente con la estabilidad económica del hogar. A partir del predominio de los ciclos de pobreza en zonas con recursos limitados, precisamente localizadas en las áreas rurales o marginales del territorio, aparece una tendencia al abandono escolar desde edades tempranas, que se destaca entre los varones, quienes acceden actividades de tipo agrícola. Si bien la educación inicial representa una inversión fundamental para el desarrollo básico del niño; todavía los encargados de las familias prefieren incorporar a sus descendientes, en forma directa, en el ciclo previo o de transición a la inserción escolar.

En consecuencia, la población sensible de una atención educativa profesional durante los períodos óptimos del desarrollo, queda condicionada para el sistema como compensación sobre el mantenimiento con dignidad de la situación económica en el hogar, lo cual podría conducir a la privación de una enseñanza de calidad en la niñez.

\section{Factores sociales relacionados con la matrícula}

En una aproximación de referencia sobre el nivel social de los hogares de proveniencia de la población infantil en los centros educativos, los recientes datos censales incluidos en el actual resumen del informe sobre el Estado de la Educación (Programa Estado de la Nación, 2013) revelan como "uno de cada tres niños de entre 0 y 6 años vive en un hogar pobre" (p. 31), una condición vulnerable que se acentúa en el tipo de hogar monoparental, lo cual puede llegar a mostrar una incidencia en las alternativas para potenciar de manera óptima las capacidades infantiles, de acuerdo con las expectativas del proceso de educación inicial, así como en la asistencia de la niñez en el sistema de enseñanza formal. 
El nivel socioeconómico de los hogares adquiere trascendencia para la reflexión en cuanto a la planificación del proyecto educativo en las diversas regiones del país. Las emergentes asociaciones familiares se circunscriben dentro de las características del desarrollo social particular de cada época. El individuo llega a convertirse en un reflejo del hogar de procedencia y, al mismo tiempo ese núcleo familiar se estructura en función de las exigencias en las transacciones del mercado globalizado. La capacidad familiar para inscribir a los hijos dentro del sistema de enseñanza formal, depende en gran medida del tipo de jefatura del hogar, sin implicar que represente una restricción, sino una condición ante las demandas del proceso educativo.

La cultura de pertenencia del estudiantado relacionada con la inserción del núcleo familiar en una determinada región, representan factores de interés para el análisis sobre las condiciones que preceden a los contextos sociales por considerar durante la previsión del proyecto educativo. "El ámbito de residencia es una variable de muy alta incidencia sobre la situación de la educación de los niños. En general, está muy asociada a la pobreza y a un bajo nivel educativo de las familias" (Itzcovich, 2010, p. 15). La realidad social de la población por atender los centros de educación inicial, refleja el apremio de gestionar oportunidades educativas efectivas, dirigidas a quienes perciben condiciones sensibles para su posible incorporación en una organización con una oferta pedagógica de calidad.

De privar al infante de experiencias favorables para su desarrollo en el hogar, por las decisiones económicas de la jefatura familiar, el entorno educativo formal podría representar un ambiente estratégico para intervenir de manera oportuna en el proceso de aprendizaje de la niñez; por la asociación entre el nivel socioeconómico con la ampliación del potencial en el conocimiento individual, de manera que "un entorno más rico y con más infraestructura durante la infancia se encuentra positivamente relacionado con una mayor habilidad cognitiva" (Mayer, López y Serván, 2008, p. 132). Sin embargo, la posibilidad de incorporar a los niños y niñas dentro del sistema de educación inicial puede figurar como un desafío para la economía de hogares en condición vulnerable.

Ofrecer alternativas a quienes requieren de programas de beneficio socio-educativo puede concretar servicios de atención infantil que abarquen a la población en condición etaria para recibir una educación especializada en las características de su proceso de aprendizaje. Los esfuerzos por emprender un proyecto educativo inclusivo han de propiciar su continua reflexión, con el propósito de asegurar la integración cultural en los diversos contextos educativos.

Existe un interés por reconocer la participación intercultural en el aula escolar. Sin embargo, en la práctica pedagógica ese beneficio se mantiene como una básica advertencia superficial en cuanto a la existencia del otro, posponiendo la inserción activa de la cultura de procedencia dentro de la implementación del currículo educativo. 


\section{Conclusiones}

En los variables contextos actuales, la información representa un elemento básico para ofrecer prácticas innovadoras que determinen el progreso de las organizaciones educativas. Al considerar la complejidad en los factores que intervienen en la conformación del proyecto educativo, se visualiza la implementación de alternativas por medio de las cuales compensar el efecto de las transformaciones del ambiente.

La conformación familiar constituye un elemento crítico sobre el proceso de gestión de la educación, puesto que implica la pertinente identificación de circunstancias que podrían conducir a aparentes procedimientos excluyentes del sistema formal. En el intento por garantizar la educación a la población en edad temprana, resulta imperante traducir en acciones toda intención proveniente de propuestas internacionales y acogida en los planes de gobierno en los diversos contextos de cada región. Dichas iniciativas resultan posibles con la planificación dirigida hacia el cambio de la situación actual mediante la implementación de planes con estrategias visionarias.

Ejercer con un liderazgo acertado la gestión de los centros de enseñanza, conduce al emprendimiento de una pedagogía ajustada a las expectativas educativas en cada zona del país, donde se logre la misión consensuada por desarrollar ante las complejas transformaciones sociales. Los datos sobre la condición en los índices de natalidad, reflejan la necesidad de atender los centros educativos mediante una gestión que considere la situación social de los núcleos familiares y la valoración que cada uno de ellos asume sobre el ingreso de sus hijos o hijas al sistema de educación.

La trascendencia del nivel socioeconómico de los hogares se advierte como fundamental para una posible reflexión en cuanto a la planificación educativa en la gestión de las organizaciones en la compleja diversidad de regiones en el sistema de educación. La articulación de acciones entre los distintos organismos gubernamentales, podría emerger como uno de los procesos para la mejora de las condiciones de gestión actuales.

Resulta fundamental el desarrollo de prácticas administrativas ejercidas con liderazgo, desde las cuales se promueva la gestión de acciones oportunas para el mejoramiento organizacional, con base en la implementación de proyectos innovadores en los que se coordinen dinámicas con el compromiso de los agentes con la misión educativa.

La proyección sobre la incorporación estudiantil representa un proceso que requiere de la fundamentación sistémica de información relevante para tomar decisiones estratégicas conducentes hacia la generación de ajustes en el sistema educativo, en consideración de los factores que podrían incidir sobre el comportamiento de la matrícula de los centros de educación inicial. 
Por las transformaciones sociales que transcurren resulta necesario fundamentar la gestión de la educación a través de un análisis sistemático, desde el cual se obtengan insumos a considerar por quienes se encargan de las políticas educativas, para examinar las opciones referentes a la promoción de aperturas para contemplar ajustes en el sistema preescolar debido a los fenómenos demográficos, familiares y sociales que podrían ejercer influencia sobre la matrícula en los centros de educación infantil.

\section{Referencias}

Castells, M. (1999). Globalización, identidad y Estado en América Latina. (Ponencia Temas de desarrollo humano sustentable). Recuperado en: http://www.desarrollohumano.cl/otraspub/Pub01/ldyest.pdf

Cerro, S. (2005). Elegir la excelencia en la gestión de un centro educativo. España: Narcea.

Delors, J. (1996). La educación encierra un tesoro. Informe a la UNESCO de la Comisión Internacional sobre la Educación para el Siglo XXI. Recuperado en: http://www.unesco.org/education/pdf/DELORS_S.PDF

Programa Estado de la Nación (2011). Tercer informe Estado de la Educación. San José, Costa Rica: Autor.

Fernández, L. (2005). Gestión por competencias. Un modelo estratégico para la dirección de recursos humanos. México: Prentice Hall.

González, S. (2011). Evaluación para la mejora de los centros educativos. España: Wolters Kluvers.

Instituto Nacional de Estadística y Censo. (2012). Características sociales y demográficas. (IX Censo Nacional de Población). San José, Costa Rica: Autor.

Itzcovich, G. (2010). Tendencias recientes del nivel inicial: un análisis estadístico de la situación argentina. Buenos Aires: Organización de Estados Iberoamericanos para la Educación, la Ciencia y la Cultura.

Mañu, J. (2009). Manual básico de dirección escolar. Dirigir es un arte y una ciencia. España: Narcea.

Maureira, O. (2006). Dirección y eficacia escolar, una relación fundamental. Revista Electrónica Iberoamericana sobre Calidad, Eficacia y Cambio en Educación, 4(4e), pp. 1-10. Recuperado en: http://www.rinace.net/arts/vol4num4e/art1.pdf 
Mayer, D.; López, M. y Serván, E. (2008). Habilidades cognitivas: transmisión intergeneracional por niveles socioeconómicos. Estudios económicos, 23(1), pp. 29-156. Recuperado en: http://www.redalyc.org/ pdf/597/59723105.pdf

Ministerio de Educación Pública. (2002). Plan de Acción de Educación 2003-2015. San José, Costa Rica: Autor.

Ministerio de Educación Pública. (2009). Normas y procedimientos para la autorización de nuevos servicios de educación preescolar. San José, Costa Rica: Autor.

Pedone, C. (2006). Los cambios familiares y educativos en los actuales contextos migratorios ecuatorianos: Una perspectiva transatlántica. Athenea Digital, 10, pp. 154-171. Recuperado en: http://www.raco. cat/index.php/Athenea/article/view/53141

Pérez, N. (2002). Funcionamiento, diagnóstico, evaluación y propuesta administrativa en la educación preescolar. (Tesina de Licenciatura). Universidad Pedagógica Nacional. Recuperado en: dialnet. unirioja.es/descarga/articulo/2133788.pdf

Programa Estado de la Nación en Desarrollo Humano Sostenible. (2013). Resumen cuarto Informe Estado de la Educación. San José, Costa Rica: Editorama.

Quilodrán, J. y Castro, T. (mayo-agosto 2009). Nuevas dinámicas familiares. Estudios demográficos y urbanos, 24(2), pp. 283-291. Recuperado en: http://www.redalyc.org/pdf/312/31221534001.pdf

Robbins, S. y Coulter, M. (2000). Administración. México: Pearson Educación.

Romero, C. (2004). La escuela media en la sociedad del conocimiento. Argentina: Ediciones Novedades Educativas.

Rosero, L. y Jiménez, P. (2012). Retos y oportunidades del cambio demográfico para la política fiscal. San José, Costa Rica: Centro Centroamericano de Población de la Universidad de Costa Rica.

Ruiz, C. (2002). Propuestay validación de un modelo decalidadeneducación infantil.(Memoria doctoral). Universidad Complutense de Madrid. Recuperado en: http://biblioteca.ucm.es/tesis/edu/ucm-t26171.pdf 
Sime, L. (2010). Informe de desarrollo humano. Revista Educación, 37, pp. 107-114. Recuperado en: revistas. pucp.edu.pe/index.php/educacion/article/download/2554/249

Simonetta, D. (2010). La estructura escolar inhibidora del aprendizaje organizacional: experiencia derivada de caso. Revista Actualidades Investigativas en Educación, 10(1), pp. 1-21. Recuperado en: http://revista.inie.ucr.ac.cr/uploads/tx_magazine/caso.pdf 